\title{
Add-on bevacizumab can prevent early clinical deterioration and prolong survival in newly diagnosed partially resected glioblastoma patients with a poor performance status
}

\author{
This article was published in the following Dove Press journal: \\ OncoTargets and Therapy \\ 18 January 2017 \\ Number of times this article has been viewed
}

\author{
Nobuhiro Hata ${ }^{1,2}$ \\ Koji Yoshimoto' \\ Ryusuke Hatae' \\ Daisuke Kuga' \\ Yojiro Akagi' \\ Yuhei Sangatsuda' \\ Satoshi O Suzuki ${ }^{3}$ \\ Tadahisa Shono ${ }^{1,4}$ \\ Masahiro Mizoguchi, ${ }^{1,5}$ \\ Koji lihara' \\ 'Department of Neurosurgery, \\ Graduate School of Medical Sciences, \\ Kyushu University, ${ }^{2}$ Department of \\ Neurosurgery, Clinical Research \\ Institute, National Hospital \\ Organization Kyushu Medical Center, \\ ${ }^{3}$ Department of Neuropathology, \\ Graduate School of Medical Sciences, \\ Kyushu University, ${ }^{4}$ Department \\ of Neurosurgery, Harasanshin \\ Hospital, Fukuoka, ${ }^{5}$ Department of \\ Neurosurgery, Kitakyushu Municipal \\ Medical Center, Kitakyushu, Japan
}

Correspondence: Nobuhiro Hata Department of Neurosurgery, Clinical Research Institute, National Kyushu Medical Center, I-8-I Jigyohama, Chuo-ku, Fukuoka 8I 0-8563, Japan

$\mathrm{Tel}+8$ I 928520700

Fax +8I 928468485

Email hatanobu@kyumed.jp
Purpose: The AVAglio trial established the beneficial effect of add-on bevacizumab (BEV) for the treatment of newly diagnosed glioblastomas (nd-GBMs) that led to the approval of BEV for the treatment of these patients in Japan. However, the rationality of using BEV as a first-line treatment for nd-GBMs remains controversial. The purpose of this study was to analyze the outcomes of a case series of nd-GBM patients.

Patients and methods: The outcomes of 69 nd-GBM patients treated after 2006 were retrospectively analyzed. Clinical and genetic analyses were performed, and estimates of progression-free survival (PFS) and overall survival (OS) were calculated using the KaplanMeier method. Since add-on BEV therapy was only used for partially resected GBMs (pr-GBMs) after its approval in 2013, the patients were subdivided into 3 treatment groups: Type I, partial removal with temozolomide (TMZ)/BEV and concurrent radiotherapy (CCRT); Type II, partial removal with TMZ and CCRT; and Type III, gross total removal with TMZ and CCRT.

Results: The PFS rate of Type I patients was significantly higher than that of Type II patients ( $P=0.014)$, but comparable to that of Type III patients. Differences in OS rates between Type I and Type II patients were less apparent $(P=0.075)$, although the median OS of Type I patients was $\sim 8$ months higher than that of Type II patients (17.4 vs 9.8 months, respectively). The clinical deterioration rate during initial treatment was significantly $(P=0.024)$ lower in Type I than in Type II patients (7.7\% vs 47.4\%, respectively). Differences in OS rates between Type I and Type II patients with a poor performance status (PS) were significant $(P=0.017)$.

Conclusion: Our findings suggest that add-on BEV can prevent early clinical deterioration of pr-GBM patients and contribute to a prolonged survival, especially for those with a poor PS.

Keywords: bevacizumab, glioblastoma, performance status, survival, unresectable

\section{Introduction}

Glioblastoma (GBM) is a common type of brain tumor that has an especially poor prognosis. Temozolomide (TMZ) treatment and concurrent radiotherapy (CCRT) followed by maintenance with TMZ (the Stupp regimen) have been regarded as the global standard for patients with newly diagnosed GBMs (nd-GBMs). ${ }^{1}$ The AVAglio trial, ${ }^{2}$ mainly conducted in European and Asian countries, has established the beneficial effect of adding bevacizumab (BEV; a humanized monoclonal antibody targeting vascular endothelial growth factor) to the Stupp regimen for improving progression-free survival (PFS) rates in GBM patients. However, no significant improvements in overall survival (OS) rates were reported. ${ }^{2}$ In the Japanese population of the AVAglio trial, ${ }^{3}$ 
although the effect of BEV on OS was not statistically significant, the median OS was longer in the BEV-treated group compared to the placebo group. Subsequently, BEV was approved in Japan, in 2013, as a first-line treatment for nd-GBMs. Currently, Japan is the only country where BEV is routinely available for treating nd-GBMs in a clinical setting. BEV for recurrent GBMs had been approved in a limited number of countries. ${ }^{4}$ However, BEV was not approved in such countries for nd-GBMs because of controversies surrounding its clinical benefits, as demonstrated by another randomized study (the Radiation Therapy Oncology Group 0825 study), ${ }^{5}$ which failed to establish a favorable outcome of BEV treatment for OS and quality of life. The rationality of using BEV as a first-line treatment for nd-GBMs remains controversial, and there is no global consensus.

At our institute and affiliated hospitals, based on the concept that BEV treatment can contribute to the control of tumor growth through regression of the existing tumor vasculature, ${ }^{6}$ BEV has been used for the treatment of nd-GBMs when gross total removal could not be achieved. Herein, we report on a case series in which we analyze the outcomes of GBM patients and evaluate the rationality of using BEV as a firstline treatment option for patients with nd-GBMs.

\section{Patients and methods}

\section{Patients}

Since TMZ was approved in Japan in 2006, 87 patients with nd-GBMs, histologically confirmed by qualified neuropathologists according to the criteria of the World Health Organization (WHO), were registered in our brain tumor database between 2006 and 2015. Patients who had refused adjuvant treatment $(n=2)$, who had undergone immunotherapy of their own choosing $(n=2)$, who had infratentorial tumors $(n=3)$ or Type I neurofibromatosis $(n=1)$, and whose genetic status was unknown due to the lack of available tissue samples $(n=2)$ were excluded from our analysis. Genetic alterations were determined using snap-frozen tumor tissue samples obtained surgically. The present investigation was approved by the ethics committee of Kyushu University and Kyushu Medical Center. All participants had provided written informed consent. Research was conducted in accordance with the 1964 Declaration of Helsinki (as revised in Fortaleza, Brazil, October 2013). According to the results, patients with IDH1 $(\mathrm{n}=5), B R A F(\mathrm{n}=2)$, and $H 3 F 3 A(\mathrm{n}=1)$ mutations were also excluded since these are known to be genetic markers of distinct biological subgroups of GBMs. ${ }^{7,8}$ In total, 69 patients $(79.3 \%)$ were included in the final analysis to evaluate their outcomes. Since the approval of TMZ in
Japan in 2006, patients with nd-GBMs were planned to be treated with maximal safe resection and TMZ with CCRT, followed by maintenance with TMZ. After the approval of BEV in Japan in 2013, the above-mentioned adjuvant treatment regimen was applied only to those patients who had undergone gross total removal (defined as the removal of $>90 \%$ of the tumor using contrast-enhanced magnetic resonance imaging). Instead, patients who had not undergone gross total removal (ie, partial removal or biopsy) were treated with BEV plus TMZ and CCRT. Consequently, the 69 nd-GBM patients enrolled in this study were divided into 3 treatment groups: Type I, partial removal with TMZ/BEV and CCRT $(n=13)$; Type II, partial removal with TMZ and CCRT ( $\mathrm{n}=19)$; and Type III, gross total removal with TMZ and CCRT $(\mathrm{n}=37)$.

\section{BEV administration}

$\mathrm{BEV}$ was administered intravenously at a dose of $10 \mathrm{mg} / \mathrm{kg}$ body weight every 2 weeks (commencing 28 days after craniotomy or 14 days after stereotactic biopsy), followed by subsequent cycles every 2 weeks as the add-on treatment for nd-GBM patients receiving TMZ and CCRT. Maintenance treatment with BEV of the same dose commenced 4 weeks after the completion of CCRT and was performed in combination with maintenance treatment with TMZ. TMZ maintenance therapy was performed for a maximum of 24 cycles. Each physician was permitted to modify treatment intervals and/or doses, based on the patient's condition. Use of steroid was limited during the perioperative phase for the purpose of controlling the symptomatic edema.

\section{Genetic analyses}

Tissue sampling and DNA preparation were performed according to our previous study. ${ }^{9}$ The detection of hot spot mutations in the $I D H 1-2, B R A F$, and $H 3 F 3 A$ genes and promoter mutations in the TERT gene was performed according to our previous study with modifications as described in the Supplementary materials. ${ }^{10} M G M T$ methylation status was assessed using a methylation-specific polymerase chain reaction-based method as described previously. ${ }^{11}$

\section{Statistical analyses}

The main outcome measures of this study were postoperative PFS and OS, with censoring at the date of last follow-up for survivors. Disease progression was assessed according to the Response Assessment in Neuro-Oncology criteria. ${ }^{12}$ OS and PFS rates were calculated using the Kaplan-Meier method, and the between-group differences in survival distributions 
were compared using the Wilcoxon test. Multivariate Cox proportional hazards regression models were applied to estimate the hazard ratios (HRs) and $95 \%$ CIs of putative prognostic factors. All statistical analyses were conducted using JMP Pro 11 Version 11.0.0 (SAS Institute Inc., Cary, NC, USA). Probability $(P)$ values were 2-sided, and a threshold of 0.05 was used to determine statistical significance.

\section{Results}

\section{First-line treatments}

Since our study has a retrospective design, we analyzed the effect of BEV on partially resected GBM (pr-GBM) patients by comparing Type I patients with Type II patients who were used as a historical control. No significant bias of clinical or molecular factors was observed between Type I and Type II patients (Table 1). In both Type I and Type II treatment groups, the median Karnofsky performance status (KPS) score was $\sim 60$. This confirms that our case series of pr-GBM patients included a considerably higher proportion of patients with a poor performance status (PS) than previous clinical trials. ${ }^{2,5}$

Table I Clinical and molecular characteristics of newly diagnosed, partially resected glioblastoma patients $(n=32)$

\begin{tabular}{|c|c|c|c|}
\hline Characteristic & $\begin{array}{l}\text { Type I patients }{ }^{a} \\
(n=13)\end{array}$ & $\begin{array}{l}\text { Type II patients } \\
(n=19)\end{array}$ & $P$-value ${ }^{c}$ \\
\hline \multicolumn{3}{|l|}{ Age, years } & 0.298 \\
\hline Mean \pm SD & $67.2 \pm 14.1$ & $61.3 \pm 15.5$ & \\
\hline \multicolumn{3}{|l|}{ Sex, n (\%) } & 1.000 \\
\hline$M$ & $7(53.8)$ & $10(52.6)$ & \\
\hline $\mathrm{F}$ & $6(46.2)$ & $9(47.4)$ & \\
\hline \multicolumn{3}{|l|}{ KPS, points } & 0.985 \\
\hline Mean \pm SD & $62.3 \pm 18.5$ & $62.5 \pm 10.9$ & \\
\hline \multicolumn{3}{|c|}{ Tumor localization, n (\%) } & 1.000 \\
\hline Deep & $6(46.2)$ & $9(47.4)$ & \\
\hline Superficial & $7(53.8)$ & $10(52.6)$ & \\
\hline \multicolumn{3}{|c|}{ Maximum tumor diameter, $\mathrm{mm}$} & 0.483 \\
\hline Mean \pm SD & $51.9 \pm 20.9$ & $57.5 \pm 21.1$ & \\
\hline \multicolumn{3}{|c|}{ Extent of resection, $\mathrm{n}(\%)$} & 0.666 \\
\hline Partial & $10(72.7)$ & $16(84.2)$ & \\
\hline Biopsy & $3(18.2)$ & $3(15.8)$ & \\
\hline \multicolumn{3}{|c|}{ MGMT status, $\mathrm{n}(\%)$} & 0.720 \\
\hline Methylated & $7(53.8)$ & $8(42.1)$ & \\
\hline Unmethylated & $6(46.2)$ & II (57.9) & \\
\hline \multicolumn{3}{|l|}{ TERT status, $\mathrm{n}(\%)$} & 1.000 \\
\hline Mutant & $9(69.2)$ & $12(63.2)$ & \\
\hline Wild-type & $4(30.8)$ & $7(36.8)$ & \\
\hline
\end{tabular}

Notes: ${ }^{\mathrm{T} T y p e}$ I patients underwent partial removal with TMZ/BEV and CCRT. 'Type II patients underwent partial removal with TMZ and CCRT. 'Student's $t$-test was performed for continuous variables, and Fisher's exact test was performed for categorical variables.

Abbreviations: BEV, bevacizumab; CCRT, concurrent radiotherapy; F, female; KPS, Karnofsky performance status; M, male; SD, standard deviation; TMZ, temozolomide.
Treatments after progression of pr-GBMs

In the Type I treatment group, with the exception of 2 patients who died of unrelated diseases without recurrence, disease progression was observed in 9 of the remaining 11 patients. Of these 9 patients, 4 patients continued BEV treatment and 1 patient received reirradiation to the recurrent lesion with concurrent BEV treatment. The remaining 4 patients were treated with best supportive care. In the Type II treatment group, of the 18 patients with disease progression (with the exception of 1 patient who died of an unrelated disease without recurrence), 2 patients continued TMZ treatment, 10 patients were treated with best supportive care, and 3 patients underwent resection of the recurrent lesion, followed by maintenance with TMZ (including 1 patient who was treated with Cyber-Knife Radiosurgery after resection). The remaining 3 patients received BEV treatment (these recurrences occurred after Japanese approval of BEV in 2013). In summary, after disease progression in a total of 27 pr-GBM patients (Type I and Type II treatment groups combined), 14 (51.8\%) patients did not receive second-line therapy, and the ratios of these patients were comparable between the Type I and Type II treatment groups (44.4\% vs $55.6 \%$, 2-sided Fisher's exact test, $P=0.71$ ).

\section{Survival analyses}

To verify the rationality of using BEV as a treatment for pr-GBMs, we evaluated the outcome of Type I patients by comparing them with Type II patients. The PFS rate of Type I patients was significantly higher than that of Type II patients ( $P=0.014$; Figure 1$)$, but comparable to that of Type III patients. The median PFS rates across the 3 groups were 10.0, 2.6, and 8.5 months, respectively. According to the multivariate Cox proportional hazards model of PFS, BEV treatment proved to be a significant prognostic factor $(P=0.022)$ among the clinical and molecular markers analyzed (Table 2). Moreover, the differences in OS rates between Type I and Type II patients were not found to be statistically significant $(P=0.075)$, although the median OS of Type I patients was $\sim 8$ months higher than that of Type II patients (17.4 vs 9.8 months, respectively; Figure 2). The multivariate Cox proportional hazards model showed BEV as a marginal significant prognostic factor $(P=0.054)$ among the clinical and molecular markers analyzed (Table 3 ).

To elucidate the benefits of BEV for the maintenance of PS in patients with pr-GBMs, we evaluated the differences in KPS scores before and after the initial treatment for Type I and II patients. The rate of clinical deterioration 


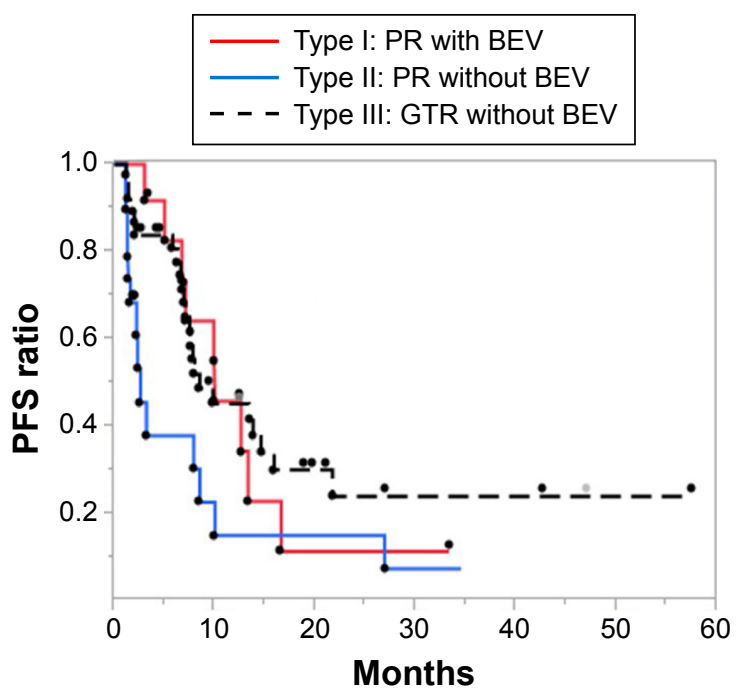

Figure I Kaplan-Meier estimates of PFS rate in newly diagnosed glioblastoma patients $(n=69)$ stratified according to treatment type.

Notes: Type I ( $n=13$; partial removal with TMZ/BEV and CCRT), Type II ( $n=19$; partial removal with TMZ and CCRT), and Type III patients ( $n=37$; gross total removal with TMZ and CCRT) are represented by the red, blue, and black (dotted) lines, respectively.

Abbreviations: BEV, bevacizumab; CCRT, concurrent radiotherapy; GTR, grosstotal removal; PFS, progression-free survival; PR, partial removal; TMZ, temozolomide.

(defined as a $\geq 20$ point reduction in KPS scores, according to the AVAglio study ${ }^{2}$ ) during the initial treatment was significantly lower (2-sided Fisher's exact test, $P=0.024$ ) in Type I patients $(\mathrm{n}=1 ; 7.7 \%)$ than in Type II patients ( $\mathrm{n}=9 ; 47.4 \%)$. This suggests that add-on BEV contributes to the prevention of early clinical deterioration in Type I patients.

We hypothesized that the benefit of BEV treatment (ie, prevention of early clinical deterioration) may contribute more favorably to the prolongation of survival in patients with a poor PS. To investigate this, we evaluated the survival outcomes of patients $(\mathrm{n}=36)$ with a poor PS, defined as having a KPS score of $\leq 70$ that is equivalent to a PS

Table 2 Prognostic factors of PFS in newly diagnosed, partially resected glioblastoma patients $(n=32)$

\begin{tabular}{lll}
\hline Factors & PFS \\
\cline { 2 - 3 } & HR (95\% CI) & P-value \\
\hline Without BEV & $3.47(1.19-11.4)$ & $0.022^{*}$ \\
Older age $(>65$ years $)$ & $1.28(0.46-3.60)$ & 0.630 \\
Low KPS score $(\leq 70)$ & $1.03(0.31-3.8 I)$ & 0.968 \\
Deep localization & $1.02(0.33-3.18)$ & 0.975 \\
Dissemination & $5.05(1.48-19.4)$ & $0.009^{*}$ \\
Large tumor diameter $(>70 \mathrm{~mm})$ & $0.92(0.27-2.96)$ & 0.885 \\
Unmethylated MGMT status & $3.79(1.10-13.7)$ & $0.035^{*}$ \\
TERT promoter mutation & $0.97(0.27-3.63)$ & 0.964 \\
\hline
\end{tabular}

Note: $* P<0.05$.

Abbreviations: BEV, bevacizumab; HR, hazard ratio; KPS, Karnofsky performance status; PFS, progression-free survival.

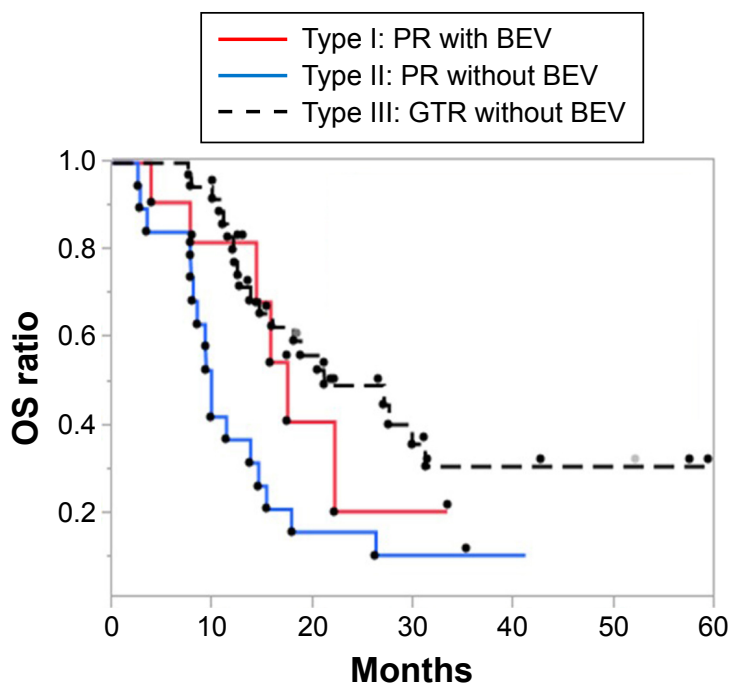

Figure 2 Kaplan-Meier estimates of OS rate in newly diagnosed glioblastoma patients $(n=69)$ stratified according to treatment type.

Notes: Type I ( $n=13$; partial removal with TMZ/BEV and CCRT), Type II ( $n=19$; partial removal with TMZ and CCRT), and Type III patients ( $n=37$; gross total removal with TMZ and CCRT) are represented by the red, blue, and black (dotted) lines, respectively.

Abbreviations: BEV, bevacizumab; CCRT, concurrent radiotherapy; GTR, grosstotal removal; OS, overall survival; PR, partial removal; TMZ, temozolomide.

of $\geq 2$ according to the criteria of the WHO, indicating that patients cannot carry out normal daily activities. The OS rate of Type I patients $(\mathrm{n}=10)$ was significantly higher than that of Type II patients ( $\mathrm{n}=11 ; P=0.017$; Figure 3$)$, but comparable to that of Type III patients $(\mathrm{n}=15)$. According to the multivariate Cox proportional hazards model of OS in patients with a poor PS, BEV treatment appeared to be a significant prognostic factor $(P=0.026)$ among the clinical and molecular markers analyzed (Table 4). These findings suggest that the contribution of add-on BEV to the prolongation of OS was more apparent in patients with a poor PS.

Table 3 Prognostic factors of OS in newly diagnosed, partially resected glioblastoma patients $(n=32)$

\begin{tabular}{lll}
\hline Factors & OS \\
\cline { 2 - 3 } & HR (95\% CI) & P-value \\
\hline Without BEV & $2.92(0.98-9.94)$ & $0.054^{* *}$ \\
Older age $(>65$ years) & $1.79(0.61-5.34)$ & 0.284 \\
Low KPS score $(\leq 70)$ & $1.89(0.67-5.83)$ & 0.235 \\
Deep localization & $1.48(0.49-4.80)$ & 0.490 \\
Dissemination & $3.10(0.97-10.4)$ & $0.056^{* *}$ \\
Large tumor diameter $(>70 \mathrm{~mm})$ & $1.51(0.48-4.74)$ & 0.469 \\
Unmethylated MGMT status & $7.79(1.81-37.1)$ & $0.005^{*}$ \\
TERT promoter mutation & $0.57(0.19-1.72)$ & 0.310 \\
\hline
\end{tabular}

Notes: $* P<0.05 ; * * P<0.10$.

Abbreviations: BEV, bevacizumab; HR, hazard ratio; KPS, Karnofsky performance status; OS, overall survival. 


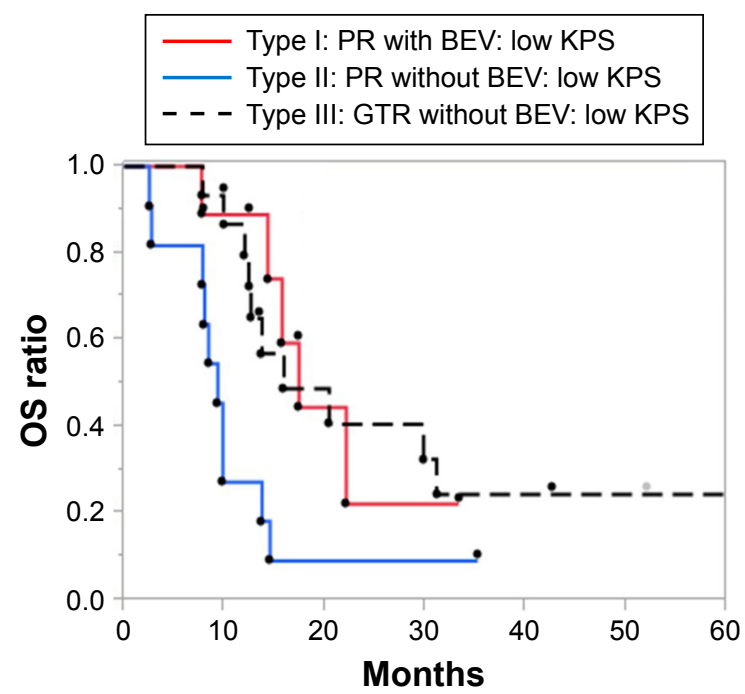

Figure 3 Kaplan-Meier estimates of OS rate in newly diagnosed glioblastoma patients with a poor performance status $(n=36)$ stratified according to treatment type. Notes: Type I ( $n=10$; partial removal with TMZ/BEV and CCRT), Type II $(n=1$; partial removal with TMZ and CCRT), and Type III patients $(n=15$; gross total removal with TMZ and CCRT) are represented by the red, blue, and black (dotted) lines, respectively.

Abbreviations: BEV, bevacizumab; CCRT, concurrent radiotherapy; GTR, grosstotal removal; OS, overall survival; PR, partial removal; TMZ, temozolomide.

\section{BEV toxicity}

During the course of CCRT, the BEV-related toxicities that led to treatment being discontinued included Grade II neutropenia and thrombocytopenia that were each observed in a single patient. With the exception of myelosuppression, discontinuation of maintenance treatment with BEV due to treatment-related toxicities occurred in 3 Type I patients, including 2 patients with deep vein thrombosis (DVT) and 1 patient who presented with gradually progressive brain atrophy after 1 year of maintenance with BEV.

\section{Discussion}

In our retrospective analysis of PFS, add-on BEV to the initial treatment of patients with pr-GBMs was associated

Table 4 Prognostic factors of OS in newly diagnosed, partially resected glioblastoma patients with a poor performance status $(\mathrm{n}=2 \mathrm{I})$

\begin{tabular}{lll}
\hline Factor & OS \\
\cline { 2 - 3 } & HR $(95 \% \mathrm{CI})$ & P-value \\
\hline Without BEV & $4.2 \mathrm{I}(\mathrm{I} .18-18.4)$ & $0.026^{*}$ \\
Older age $(>65$ years $)$ & $2.89(0.46-19.8)$ & $0.25 \mathrm{I}$ \\
Deep localization & $0.75(0.15-4.08)$ & 0.732 \\
Dissemination & $5.89(1.38-31.3)$ & $0.016^{*}$ \\
Large tumor diameter $(>70 \mathrm{~mm})$ & $1.12(0.24-4.94)$ & 0.883 \\
Unmethylated MGMT status & $\mathrm{II} .0(\mathrm{I} .44-97.0)$ & $0.02 I^{*}$ \\
TERT promoter mutation & $0.54(0.14-2.28)$ & 0.387 \\
\hline
\end{tabular}

Note: $* P<0.05$

Abbreviations: BEV, bevacizumab; HR, hazard ratio; OS, overall survival. with a better outcome and significantly lower HR compared to TMZ treatment alone, as similarly shown in previous Phase III clinical trials. ${ }^{2,5}$ We also demonstrated that the PS is well maintained in patients treated with BEV compared to patients treated without BEV. Furthermore, in an analysis of OS, we showed a favorable outcome of add-on BEV treatment for patients with pr-GBMs, especially those with a poor PS. Taken together, our findings from this retrospective study suggest that add-on BEV treatment may contribute to the prevention of early clinical deterioration and lead to a prolongation of OS more favorably in pr-GBM patients with a poor PS. Our result might also support the rescue use of BEV for patients whose condition deteriorates.

Previous large clinical trials ${ }^{2,5}$ have failed to demonstrate a prolongation of OS in nd-GBM patients treated with add-on BEV in addition to the standard initial treatment. With respect to pr-GBM patients as well, a beneficial effect was not demonstrated in a subanalysis of data from the AVAglio trial, ${ }^{2}$ which revealed no effect of BEV treatment on the outcomes of patients who underwent partial resections; ${ }^{13}$ thus, revealing a discrepancy between previous findings and the findings of our present study. The most notable difference between the backgrounds of the patients in our study compared to the patients of the aforementioned clinical trials is their PS. In the AVAglio trial, ${ }^{2}$ a WHO PS of $\leq 2$ (equivalent to a KPS score of $\geq 60$ ) was an inclusion criterion and $\sim 50 \%$ of the enrolled patients maintained a WHO PS of 0 (equivalent to a KPS score of 90-100). Conversely, KPS scores in $\sim 50 \%$ of pr-GBM patients in the present study were $\leq 70$. In real clinical settings, as demonstrated in the present study, general GBM populations include considerably more patients with a poor PS, in contrast to the AVAglio trial. ${ }^{14}$ The strict selection criteria for participation in clinical trials can lead to such specificity of the enrolled patients. ${ }^{15}$

The beneficial effect of add-on BEV for patients with a poor PS was suggested even in a subanalysis of the AVAglio trial $^{2}$ that revealed lower HRs for PFS in patients with a WHO PS of 1-2 compared to patients with a WHO PS of 0 . Another exploratory analysis of the AVAglio trial ${ }^{15}$ revealed that $\mathrm{BEV}$ prolonged the OS of patients who did not receive second-line therapy after disease progression. The primary purpose of this exploratory analysis was to evaluate the impact of poststudy crossover. However, the authors ${ }^{15}$ took into consideration the fact that a greater proportion of patients with poor prognostic features may be included in this analysis. In the present study, $51.7 \%$ of pr-GBM patients did not receive second-line therapy after progression, and the ratio was considerably higher than that of the AVAglio trial ${ }^{15}$ 
(24.4\%). These findings suggest that pr-GBM patients are less likely to receive second-line therapy after progression. That is, $\mathrm{BEV}$ is used as a treatment option for recurrent tumors would not contribute reliably to the favorable outcome of prGBM patients. Taken together, it is assumed that BEV would be more beneficial for the treatment of nd-GBM patients with a progressive clinical course (eg, pr-GBM patients with a poor PS) than for the usual patient populations enrolled in clinical trials.

Our study has several limitations, including its nonrandomized retrospective design and the small number of enrolled patients from a limited number of institutions, and using a historical control group that may include a considerable proportion of patients who might not have received BEV as salvage treatment. Use of historical controls might be problematic because insights and techniques of treatment for glioma are changing over time. To obtain more credible results and to elucidate further the precise benefits of BEV treatment in real clinical settings, future clinical trials, without limiting criteria (eg, PS), are warranted.

In our case series, DVT was the only apparent adverse event that resulted in the discontinuation of BEV treatment. In the safety data from the AVAglio trial, ${ }^{16}$ the incidence of arterial thromboembolic events was higher in the BEV-treated group compared to the placebo control group (5.9\% vs $1.6 \%$, respectively). A meta-analysis ${ }^{17}$ revealed a trend toward a significant association between BEV treatment and the risk of developing DVT and pulmonary embolisms. Another complication suspected to be associated with the discontinuation of BEV treatment was brain atrophy. A previous study ${ }^{18}$ reported that prolonged $\mathrm{BEV}$ treatment was associated with brain atrophy. In a laboratory investigation, ${ }^{19}$ long-term BEV treatment was found to be associated with a reduction in the dendritic length of hippocampal neurons. Nonetheless, our treatment approach can contribute to relatively long-term survival for patients with pr-GBMs (ie, it should lead to more frequent adverse events derived from prolonged treatment). Although a previous report ${ }^{20}$ suggested that discontinuation of BEV treatment could lead to a rebound phenomenon, recent studies ${ }^{21,22}$ revealed that treatment intervals of $\mathrm{BEV}$ were not associated with a poor outcome. For the purpose of maximizing the clinical benefits to patients, further efforts to address the appropriate timings of BEV discontinuation will be required.

\section{Acknowledgments}

The authors wish to thank Dr Hiroshi Muratani (Ando Hospital, Fukuoka, Japan) for his provision of clinical data and Ms Fumie Doi (Kyushu University) for her technical assistance. This work was supported by a Japanese Society for the Promotion of Science Grants-in-Aid for Scientific Research (KAKENHI) Award (Grant No 26462185, 25293311, 15K15529 and 16K10779).

\section{Disclosure}

The authors report no conflicts of interest in this work.

\section{References}

1. Stupp R, Mason WP, van den Bent MJ, et al. Radiotherapy plus concomitant and adjuvant temozolomide for glioblastoma. $N$ Engl J Med. 2005;352(10):987-996.

2. Chinot OL, Wick W, Mason W, et al. Bevacizumab plus radiotherapytemozolomide for newly diagnosed glioblastoma. N Engl J Med. 2014; 370(8):709-722.

3. Takano S, Ishikawa E, Nakai K, et al. Bevacizumab in Japanese patients with malignant glioma: from basic research to clinical trial. Onco Targets Ther. 2014;7:1551-1562.

4. Cohen MH, Shen YL, Keegan P, Pazdur R. FDA drug approval summary: bevacizumab (avastin) as treatment of recurrent glioblastoma multiforme. Oncologist. 2009;14(11):1131-1138.

5. Gilbert MR, Dignam JJ, Armstrong TS, et al. A randomized trial of bevacizumab for newly diagnosed glioblastoma. N Engl J Med. 2014; 370(8):699-708.

6. O'Connor JP, Carano RA, Clamp AR, et al. Quantifying antivascular effects of monoclonal antibodies to vascular endothelial growth factor: insights from imaging. Clin Cancer Res. 2009;15(21):6674-6682.

7. Schindler G, Capper D, Meyer J, et al. Analysis of BRAF V600E mutation in 1,320 nervous system tumors reveals high mutation frequencies in pleomorphic xanthoastrocytoma, ganglioglioma and extra-cerebellar pilocytic astrocytoma. Acta Neuropathol. 2011;121(3):397-405.

8. Sturm D, Witt H, Hovestadt V, et al. Hotspot mutations in H3F3A and IDH1 define distinct epigenetic and biological subgroups of glioblastoma. Cancer Cell. 2012;22(4):425-437.

9. Hata N, Yoshimoto K, Hatae R, et al. Deferred radiotherapy and upfront procarbazine-ACNU-vincristine administration for $1 \mathrm{p} 19 \mathrm{q}$ codeleted oligodendroglial tumors is associated with favorable outcome without compromising patient performance, regardless of WHO grade. Onco Targets Ther. 2016;9:7123-7131.

10. Hatae R, Hata N, Yoshimoto K, et al. Precise detection of IDH1/2 and BRAF hotspot mutations in clinical glioma tissues by a differential calculus analysis of high-resolution melting data. PLoS One. 2016; 11(8):e0160489.

11. Araki Y, Mizoguchi M, Yoshimoto K, et al. Quantitative digital assessment of MGMT immunohistochemical expression in glioblastoma tissue. Brain Tumor Pathol. 2011;28(1):25-31.

12. Wen PY, Macdonald DR, Reardon DA, et al. Updated response assessment criteria for high-grade gliomas: response assessment in neurooncology working group. J Clin Oncol. 2010;28(11):1963-1972.

13. Sandmann T, Bourgon R, Garcia J, et al. Patients with proneural glioblastoma may derive overall survival benefit from the addition of bevacizumab to first-line radiotherapy and temozolomide: retrospective analysis of the AVAglio trial. J Clin Oncol. 2015;33(25):2735-2744.

14. Narita Y, Shibui S. Committee of Brain Tumor Registry of Japan Supported by the Japan Neurosurgical Society. Trends and outcomes in the treatment of gliomas based on data during 2001-2004 from the brain tumor registry of Japan. Neurol Med Chir (Tokyo). 2015;55(4): 286-295.

15. Chinot OL, Nishikawa R, Mason W, et al. Upfront bevacizumab may extend survival for glioblastoma patients who do not receive second-line therapy: an exploratory analysis of AVAglio. Neuro Oncol. 2016;18(9): 1313-1318. 
16. Saran F, Chinot OL, Henriksson R, et al. Bevacizumab, temozolomide, and radiotherapy for newly diagnosed glioblastoma: comprehensive safety results during and after first-line therapy. Neuro Oncol. 2016;18(7): 991-1001.

17. Li X, Huang R, Xu Z. Risk of adverse vascular events in newly diagnosed glioblastoma multiforme patients treated with bevacizumab: a systematic review and meta-analysis. Sci Rep. 2015;5:14698.

18. Bag AK, Kim H, Gao Y, et al. Prolonged treatment with bevacizumab is associated with brain atrophy: a pilot study in patients with high-grade gliomas. J Neurooncol. 2015;122(3):585-593.

19. Latzer P, Schlegel U, Theiss C. Morphological changes of cortical and hippocampal neurons after treatment with VEGF and bevacizumab. CNS Neurosci Ther. 2016;22(6):440-450.
20. Zuniga RM, Torcuator R, Jain R, et al. Rebound tumour progression after the cessation of bevacizumab therapy in patients with recurrent high-grade glioma. J Neurooncol. 2010;99(2):237-242.

21. Anderson MD, Hamza MA, Hess KR, Puduvalli VK. Implications of bevacizumab discontinuation in adults with recurrent glioblastoma. Neuro Oncol. 2014;16(6):823-828.

22. Hertenstein A, Hielscher T, Menn O, et al. Impact of tapering and discontinuation of bevacizumab in patients with progressive glioblastoma. J Neurooncol. 2016;129(3):533-539. 


\section{Supplementary materials Detection of hot spot mutations in glioblastoma tissues}

Mutation detection of the IDHI (codon 132), IDH2 (codon 172), BRAF (codon 600), and H3F3A (codons 27 and 34) genes was performed by high-resolution melt (HRM) analysis and subsequent Sanger sequencing. Primer sequences for the amplification of genomic DNA were designed using Primer3Plus $^{1}$ (http://bioinformatics.nl/cgi-bin/primer3plus/ primer3plus.cgi/). In silico polymerase chain reaction (PCR) applications (http://genome.ucsc.edu/cgi-bin/hgPcr) were used to verify the theoretical specificity of the forward and reverse primers. Details of the primer sequences and their amplicon sizes are provided in Table S1. Whole HRM reactions were prepared using $16.6 \mathrm{ng}$ of DNA, $7.47 \mathrm{pmol} / \mathrm{L}$ of each of the forward and reverse primers, and $10 \mu \mathrm{L}$ of MeltDoctor HRM Master Mix (Applied Biosystems ${ }^{\circledR}$, Tokyo, Japan) in a total volume of $20 \mu \mathrm{L}$, according to the manufacturer's protocol. An ABI 7,500 Fast Real-Time PCR System (Applied Biosystems) was used for amplification. The cycling conditions were as follows: 1) an initial denaturation step at $95^{\circ} \mathrm{C}$ for 10 minutes; 2) 40 cycles of $95^{\circ} \mathrm{C}$ for 15 seconds, followed by $60^{\circ} \mathrm{C}$ for 1 minute; and 3) a dissociation cycle of $95^{\circ} \mathrm{C}$ for 15 seconds, followed by $60^{\circ} \mathrm{C}$ for 1 minute, and $95^{\circ} \mathrm{C}$ for 15 seconds. Mutations were determined according to our previous study. ${ }^{2}$ Thereafter, HRM products were purified using ExoSap-IT (Affymetrix Inc., Santa Clara, CA, USA) for mutation genotyping. Cycle sequencing was performed using BigDye $^{\circledR}$ Terminator v3.1 Cycle Sequencing Kit (Applied Biosystems). After purification of the products, electrophoresis and analysis were conducted using an ABI PRISM ${ }^{\circledR}$ 310 Genetic Analyzer (Applied Biosystems).

PCR and sequencing of the TERT promoter were performed according to a previous study with slight modifications. ${ }^{3}$ In our study, we designed the following oligonucleotide primers using Primer3Plus: ${ }^{1} 5^{\prime}$-GGCCGATTCGACCTC TCT-3' and 5'-CAGCGCTGCCTGAAACTC-3'. PCR reactions were performed in $10 \mu \mathrm{L}$ volumes containing $\sim 20 \mathrm{ng}$ of DNA, $0.1 \mu \mathrm{L}$ of TaKaRa LA Taq ${ }^{\circledR}$ DNA polymerase (TaKaRa Bio Inc., Shiga, Japan), $5 \mu \mathrm{L}$ of $2 \times \mathrm{GC}$ Buffer I, $1.6 \mu \mathrm{L}$ of the deoxyribonucleoside triphosphate mixture $(2.5 \mathrm{mM}$ of each $\mathrm{dNTP})$, and $1.67 \mu \mathrm{L}$ of each of the primers $(2 \mu \mathrm{M})$. The cycling conditions were as follows: 1 ) an initial denaturation step at $95^{\circ} \mathrm{C}$ for 5 minutes; 2) 35 cycles of $94^{\circ} \mathrm{C}$ for 30 seconds, followed by $62^{\circ} \mathrm{C}$ for 30 seconds, and $72^{\circ} \mathrm{C}$ for 30 seconds; and 3) a final elongation step at $72^{\circ} \mathrm{C}$ for 7 minutes. The PCR products were gel purified and sequenced on an ABI $310^{\circledR}$ PRISM Genetic Analyzer (Applied Biosystems).

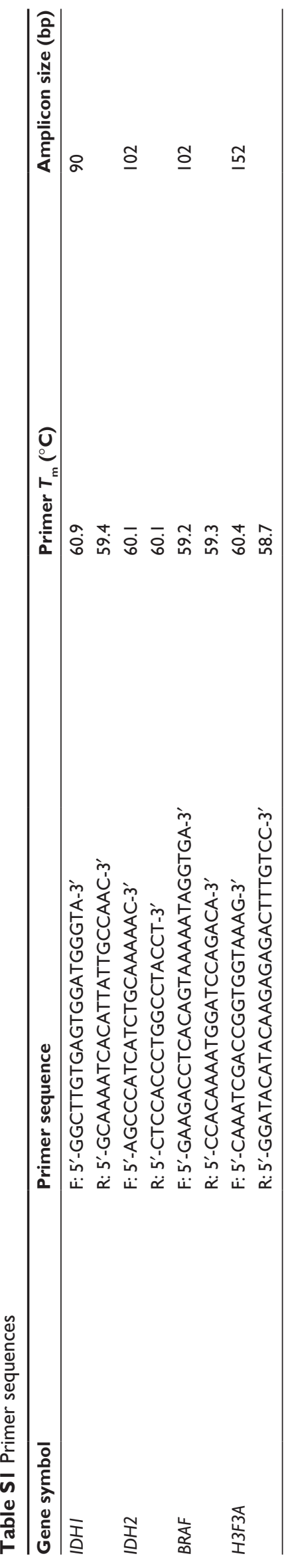




\section{References}

1. Untergasser A, Nijveen H, Rao X, Bisseling T, Geurts R, Leunissen JA. Primer3Plus, an enhanced web interface to primer3. Nucleic Acids Res. 2007;35(Web Server issue):W71-W74.

2. Hatae R, Hata N, Yoshimoto K, et al. Precise detection of IDH1/2 and BRAF hotspot mutations in clinical glioma tissues by a differential calculus analysis of high-resolution melting data. PLoS One. 2016; 11(8): 0160489.

3. Chen C, Han S, Meng L, Li Z, Zhang X, Wu A. TERT promoter mutations lead to high transcriptional activity under hypoxia and temozolomide treatment and predict poor prognosis in gliomas. PLoS One. 2014;9(6):e100297.

\section{Publish your work in this journal}

OncoTargets and Therapy is an international, peer-reviewed, open access journal focusing on the pathological basis of all cancers, potential targets for therapy and treatment protocols employed to improve the management of cancer patients. The journal also focuses on the impact of management programs and new therapeutic agents and protocols on
Dovepress

patient perspectives such as quality of life, adherence and satisfaction. The manuscript management system is completely online and includes a very quick and fair peer-review system, which is all easy to use. Visit http://www.dovepress.com/testimonials.php to read real quotes from published authors.

\footnotetext{
Submit your manuscript here: http://www.dovepress.com/oncotargets-and-therapy-journal
} 